\title{
Validation of Object Oriented Metrics for Evaluating Understandability of Data Warehouse Models
}

\author{
Jaspreeti Singh \\ Assistant Prof., CSE Dept \\ USIT, GGSIPU, Dwarka \\ Delhi-75
}

\author{
Srishti Vashishtha \\ Student, M.Tech CSE Dept. \\ USICT, GGSIPU, Dwarka \\ Delhi-75
}

\begin{abstract}
Datawarehouse has a key role in formulating strategic decisions thus it is very essential to maintain its quality. Metrics have been generally used to direct designers to develop quality data models. Numerous researchers have proposed metrics for multidimensional models for datawarehouse. These metrics are required to be empirically validated to prove their practical utility. Empirical validation of the object oriented metrics for multidimensional models for data warehouses at a conceptual level is presented in the paper. Quality attribute understandability is assessed through various combinations of metrics. Univariate and Multiple linear regression analysis have been used in this paper for computing the multidimensional models quality. The results show that these metrics may be considered as key indicators for quality of multidimensional data models.
\end{abstract}

\section{Keywords:}

Datawarehouse, Metrics, Multidimensional models, Quality attributes

\section{INTRODUCTION}

In today's world organizations need to gather, store and process huge volume of data that is needed to perform day to day operations. They are able to do this at a comparatively low cost but fail to provide quality information [6]. Inmon provided the solution of adopting datawarehouse, which is defined as "collection of subject-oriented, integrated, nonvolatile data that supports the management decision process " [4]. Datawarehouse is a type of environment for the purpose of providing strategic information for analysing, discerning trends and monitoring performance. It is essential for the organizations to ascertain the quality of the information that they are getting from the datawarehouse. The information quality in a datawarehouse is determined by the data quality, presentation quality and the quality of data model (conceptual, logical and physical data model) [3]. Our aim in this paper is to ensure quality by evaluating understandability of multidimensional models.

Structural properties have been recognised as major factors influencing quality of a software product. Metrics based on structural properties have been widely used to assess the quality attributes like understandability, maintainability; fault-proneness etc. of a software artefact [8].Our present focus is on understandability as it is the key measure of quality of datawarehouse conceptual models. There is a relationship between structural properties, cognitive complexity, understandability and external quality attributes. Structural complexity affects cognitive complexity it in turn affects analyzability, understandability \& modifiability; and these

further affect external quality [15]. Hence, the structural complexity plays a vital role while assessing the quality of a model. These complexity metrics helps to estimate the quality of information provided by the datawarehouse. A slight error in the information can cause huge losses to organizations, thus it is important to maintain the quality.

Researchers have recommended quality attributes for multidimensional models and have also proposed metrics to estimate these quality attributes. In this paper we focus on the quality attribute - understandability of multidimensional models. Metrics are the objective indicators of quality. They provide a way of measuring quality factors in a consistent and objective manner. Metrics could be useful to understand and improve software development and maintenance of projects and to maintain the quality of a system highlighting the key problematic areas. A set of metrics for data warehouse models is already presented to compute the structural complexity of a multidimensional model by Serrano et al. [15]. The author has suggested that these metrics need to be validated to ensure the practical utility of these metrics and to draw a final conclusion which may be applied in practice. Even though several quality frameworks for data models have been proposed, most of them lack valid quantitative measures to calculate the quality of conceptual data models in an objective way. This family of experiments is a significant aspect in the process of validating metrics as it is extensively accepted that only after executing a family of experiments; it is possible to develop the collective knowledge to extract constructive measurement conclusions to be applied in practice. [15] [16] Hence, in this paper, we have executed empirical validation by considering a dataset consisting of eighteen multidimensional schemas for a datawarehouse using correlation and linear regression.

The rest of this paper is structured as follows: Section 2 explains the process of metrics validation. Section 3 discusses the metrics of the object oriented models for data warehouses. Section 4 elaborates the experimental setup. Section 5 shows the result of various analysis methodologies. Sections 6 discuss about the threats to the validity of the results and limitations of the study. In the end, Section 7 summarizes the work and presents the conclusion.

\section{METRICS VALIDATION PROCESS}

Metrics Validation process has certain steps to ensure the reliability of the proposed metrics. It is necessary to follow these steps. Figure 1 presents the method we follow for the metrics proposal [3].

In this figure we have three central activities:

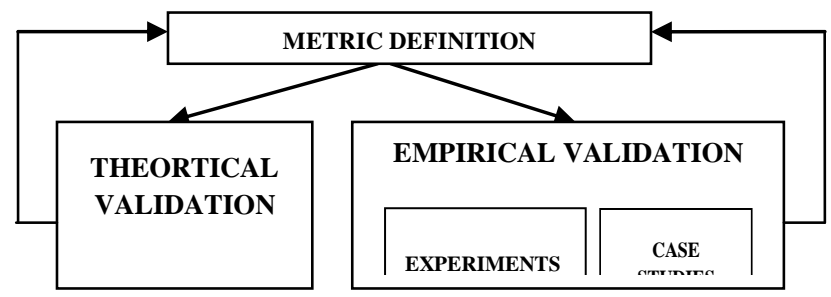


Figure 1. Steps followed in the definition and validation of metrics ref [3]

Metrics definition The initial step is the proposal of metrics. This definition is in accordance to the specific characteristics of the system we want to evaluate and the experience of designers of these systems. [3]

Theoretical validation The next step is the formal validation of the metrics. The formal validation assists us to know when and how to apply the metrics. There are two major tendencies in metrics validation: the frameworks based on axiomatic approaches [17] [2] and the ones based on the measurement theory [18]. The power of measurement theory is the formulation of empirical conditions from which we can derive hypothesis of reality. The final information when applying these kind of frameworks is to know to which scale a metric pertains and based on this information we can identify which statistics and which transformations can be performed on the metric.

Empirical validation. The objective of this step is to prove the practical utility of the proposed metrics. Although there are many ways of performing this step, we can divide the empirical validation into experimentation and case studies [1] [3].

As shown in Fig. 1, the process of defining and validating metrics is evolutionary and iterative. Metrics could be redefined or discarded based on the feedback from theoretical or empirical validations. The aim behind the empirical validation is to prove the practical utility of the proposed metrics. Thus empirical validation is vital for the success of any project.

\section{METRICS FOR}

\section{MULTIDIMENSIONAL MODEL}

The metrics proposed by Serrano et al. [15] for the star level is composed of a fact class together with all the dimension classes and associated base classes. The proposed metrics are simple, which is a desirable property of software metrics [5]. These metrics focus on the star level metrics of star schema of data warehouse conceptual model. The metrics are as follows [15]:

1. $\quad \mathbf{N D C ( S )}$ :Number of dimension classes of the star $\mathrm{S} \quad$ (equal to the number of aggregation relationships)

2. $\quad$ NBC(S) : Number of base classes of the star $S$

3. $\quad \mathbf{N C}(\mathbf{S})$ : Total number of classes of the star $\mathrm{S}$

$\mathrm{NC}(\mathbf{S})=\mathrm{NDC}(\mathrm{S})+\mathrm{NBC}(\mathrm{S})+1$

4. $\quad \mathbf{R B C}(\mathbf{S})$ : Ratio of base classes. Number of base classes per dimension class of star $S$

5. NAFC(S) : Number of FA attributes of the fact class of the star S NADC(S): Number of D and DA attributes of the dimension classes of the star $\mathrm{S}$

6. NABC(S) : Number of D and DA attributes of the base classes of the star $\mathrm{S}$

7. $\quad \mathrm{NA}(\mathrm{S})$ Total number of FA, D and DA attributes of the star $S$
$\mathrm{NA}(\mathrm{S})=\mathrm{NAFC}(\mathrm{S})+\mathrm{NADC}(\mathrm{S})+\mathrm{NABC}(\mathrm{S})$

8. $\mathrm{NH}(\mathrm{S})$ : Number of hierarchy relationships of the star $\mathrm{S}$

9. $\operatorname{DHP}(\mathrm{S})$ : Maximum depth of the hierarchy relationships of the star $S$

10. $\operatorname{RSA}(\mathrm{S})$ : Ratio of attributes of the star S. Number of attributes FA divided by the number of $\mathrm{D}$ and DA attributes, where FA is 'Fact Attribute': Attributes of this stereotype represent attributes of Fact classes in a MD model. D is 'Descriptor Attribute': Attributes of this stereotype represent descriptor attributes of dimension or base classes in a MD model. DA is 'Dimension Attribute': Attributes of this stereotype represent attributes of dimension or base classes in a MD model.

\section{EXPERIMENTAL SETUP}

\subsection{Aim}

The goal of the experiment is to analyze the metrics of multidimensional models for the purpose of computing them with respect to the data warehouse quality attributes i.e. understandability.

\subsection{Schemas}

Eighteen multidimensional data warehouse schemas were used for performing the experiment. Schemas with different metrics values are considered. Metrics values for all the eighteen schemas are given in Table1. All the schemas were taken from different domains.

\subsection{Subjects}

Total of twenty five M.Tech. students from Guru Gobind Singh Indraprastha University, New Delhi participated in the experiment as subjects.

\subsection{Hypothesis}

Null hypothesis $\mathrm{H}_{0 \text { : }}$ There is no noteworthy correlation between the metrics and the understandability of the datawarehouse conceptual models.

Alternative hypothesis $\mathrm{H}_{01}$ : $\neg \mathrm{H}_{11}$ There is a significant relation between the metrics and the understandability of the data warehouse conceptual models.

\subsection{Collected Data}

In this paper we have considered one dependent variable, Understandability: It is measured as the time taken by each subject to perform the tasks of the experimental test. The independent variables used in this experiment are the metrics, those variables for which the effect should be calculated. The experimental task consists of understanding a schema and answering the questions based on the schema. Understanding time varies according to the complexity of the schemas and understandability level of the subject. Table 2 represents the collected data (understanding time) of all the subjects for each schema.

Table 1 Values of metrics for the schemas

\begin{tabular}{|c|c|c|c|c|c|c|c|c|c|c|c|c|}
\hline & NDC & NBC & $\mathrm{NC}$ & RBC & NAFC & NADC & NABC & NA & NH & DHP & RSA & $\begin{array}{c}\text { Understandi } \\
\text { ng time }\end{array}$ \\
\hline SC01 & 4 & 9 & 2.25 & 3 & 11 & 15 & 29 & 4 & 3 & 3 & 0.11 & 65.76 \\
\hline SC02 & 5 & 17 & 22 & 3.4 & 7 & 22 & 26 & 55 & 5 & 4 & 0.14 & 79.52 \\
\hline SC03 & 6 & 9 & 15 & 1.5 & 5 & 18 & 23 & 46 & 4 & 2 & 0.12 & 74.60 \\
\hline
\end{tabular}




\begin{tabular}{|l|c|c|c|c|c|c|c|c|c|c|c|c|}
\hline SC04 & 7 & 15 & 22 & 2.14 & 5 & 30 & 19 & 54 & 3 & 3 & 0.10 & 79.40 \\
\hline SC05 & 6 & 9 & 15 & 1.5 & 4 & 17 & 23 & 44 & 4 & 3 & 0.10 & 73.20 \\
\hline SC06 & 3 & 10 & 13 & 3.33 & 4 & 9 & 8 & 21 & 2 & 2 & 0.23 & 63.10 \\
\hline SC07 & 5 & 8 & 13 & 1.3 & 3 & 23 & 20 & 46 & 4 & 3 & 0.06 & 64.60 \\
\hline SC08 & 4 & 9 & 13 & 2.25 & 4 & 20 & 17 & 41 & 3 & 3 & 0.10 & 62.40 \\
\hline SC09 & 6 & 13 & 19 & 2.16 & 5 & 26 & 24 & 55 & 4 & 5 & 0.10 & 78.30 \\
\hline SC10 & 4 & 13 & 17 & 3.25 & 5 & 19 & 17 & 41 & 3 & 3 & 0.13 & 76.80 \\
\hline SC11 & 3 & 8 & 11 & 2.66 & 4 & 13 & 8 & 25 & 2 & 2 & 0.19 & 63.24 \\
\hline SC12 & 5 & 15 & 20 & 3 & 3 & 24 & 20 & 47 & 4 & 5 & 0.06 & 79.00 \\
\hline SC13 & 4 & 4 & 8 & 1 & 1 & 23 & 12 & 36 & 2 & 3 & 0.02 & 56.18 \\
\hline SC14 & 4 & 9 & 13 & 2.25 & 1 & 20 & 11 & 32 & 2 & 3 & 0.03 & 64.44 \\
\hline SC15 & 6 & 14 & 20 & 3.33 & 5 & 18 & 26 & 47 & 4 & 5 & 0.11 & 77.00 \\
\hline SC16 & 8 & 14 & 22 & 1.4 & 10 & 30 & 12 & 52 & 6 & 4 & 0.23 & 80.00 \\
\hline SC17 & 4 & 6 & 10 & 1.5 & 3 & 15 & 6 & 24 & 3 & 2 & 0.14 & 58.00 \\
\hline SC18 & 5 & 7 & 12 & 1.4 & 4 & 20 & 8 & 32 & 4 & 2 & 0.14 & 64.80 \\
\hline
\end{tabular}

Table 2 Understanding time (in seconds) of schemas for each subject

\begin{tabular}{|c|c|c|c|c|c|c|c|c|c|c|c|c|c|}
\hline & Sub1 & Sub2 & Sub3 & Sub4 & Sub5 & Sub6 & Sub7 & Sub8 & Sub9 & Sub10 & Sub11 & Sub12 & Sub13 \\
\hline SC01 & 65 & 55 & 59 & 72 & 79 & 63 & 69 & 71 & 61 & 64 & 67 & 66 & 65 \\
\hline SC02 & 58 & 68 & 76 & 100 & 96 & 78 & 80 & 74 & 81 & 79 & 99 & 74 & 78 \\
\hline SC03 & 90 & 61 & 93 & 66 & 60 & 73 & 79 & 70 & 81 & 75 & 80 & 71 & 69 \\
\hline SC04 & 81 & 69 & 77 & 91 & 71 & 80 & 75 & 86 & 69 & 72 & 87 & 78 & 76 \\
\hline SC05 & 60 & 48 & 54 & 61 & 69 & 57 & 72 & 70 & 64 & 61 & 79 & 68 & 74 \\
\hline SC06 & 52 & 56 & 65 & 85 & 53 & 62 & 68 & 57 & 49 & 66 & 72 & 65 & 63 \\
\hline SC07 & 64 & 71 & 69 & 57 & 79 & 59 & 63 & 66 & 72 & 48 & 60 & 57 & 53 \\
\hline SC08 & 62 & 72 & 55 & 69 & 74 & 60 & 59 & 65 & 59 & 54 & 61 & 63 & 59 \\
\hline SC09 & 66 & 76 & 72 & 78 & 94 & 77 & 91 & 86 & 71 & 85 & 80 & 91 & 82 \\
\hline SC10 & 66 & 95 & 63 & 87 & 94 & 78 & 73 & 86 & 72 & 77 & 79 & 64 & 71 \\
\hline SC11 & 78 & 50 & 77 & 60 & 57 & 63 & 61 & 71 & 57 & 74 & 63 & 55 & 51 \\
\hline SC12 & 82 & 73 & 95 & 69 & 64 & 84 & 89 & 66 & 65 & 83 & 87 & 94 & 76 \\
\hline SC13 & 65 & 54 & 51 & 72 & 46 & 53 & 57 & 56 & 50 & 60 & 52 & 49 & 57 \\
\hline SC14 & 67 & 58 & 63 & 78 & 58 & 65 & 69 & 57 & 60 & 75 & 64 & 60 & 70 \\
\hline SC15 & 76 & 83 & 71 & 79 & 72 & 69 & 78 & 65 & 70 & 79 & 81 & 73 & 68 \\
\hline SC16 & 84 & 78 & 92 & 67 & 70 & 65 & 82 & 78 & 87 & 68 & 75 & 86 & 77 \\
\hline SC17 & 50 & 59 & 52 & 78 & 51 & 54 & 61 & 54 & 62 & 52 & 61 & 66 & 59 \\
\hline \multirow[t]{2}{*}{ SC18 } & 67 & 55 & 72 & 68 & 64 & 77 & 69 & 54 & 63 & 59 & 61 & 58 & 54 \\
\hline & Sub14 & Sub15 & Sub16 & Sub17 & Sub1 & & ub19 & Sub20 & Sub21 & Sub22 & Sub23 & Sub24 & Sub25 \\
\hline SC01 & 64 & 69 & 61 & 70 & 64 & & 63 & 68 & 66 & 61 & 69 & 70 & 63 \\
\hline SC02 & 76 & 85 & 71 & 92 & 82 & & 74 & 79 & 69 & 80 & 65 & 95 & 79 \\
\hline SC03 & 71 & 76 & 82 & 71 & 82 & & 67 & 90 & 66 & 72 & 69 & 80 & 71 \\
\hline SC04 & 81 & 73 & 95 & 82 & 72 & & 86 & 73 & 96 & 76 & 78 & 71 & 90 \\
\hline SC05 & 77 & 86 & 90 & 72 & 89 & & 79 & 75 & 92 & 70 & 89 & 78 & 96 \\
\hline SC06 & 64 & 68 & 57 & 76 & 60 & & 51 & 63 & 57 & 62 & 69 & 61 & 75 \\
\hline SC07 & 59 & 61 & 74 & 63 & 68 & & 50 & 65 & 80 & 61 & 59 & 77 & 81 \\
\hline SC08 & 54 & 65 & 63 & 52 & 64 & & 56 & 65 & 75 & 62 & 71 & 58 & 65 \\
\hline SC09 & 61 & 72 & 80 & 78 & 91 & & 67 & 76 & 65 & 80 & 73 & 85 & 81 \\
\hline SC10 & 68 & 66 & 70 & 74 & 80 & & 77 & 65 & 80 & 87 & 69 & 97 & 84 \\
\hline SC11 & 72 & 64 & 51 & 59 & 66 & & 67 & 75 & 59 & 55 & 61 & 66 & 69 \\
\hline SC12 & 62 & 89 & 77 & 85 & 88 & & 80 & 89 & 74 & 65 & 93 & 68 & 79 \\
\hline SC13 & 69 & 50 & 52 & 53 & 59 & & 60 & 55 & 46 & 63 & 57 & 58 & 60 \\
\hline SC14 & 77 & 55 & 62 & 71 & 78 & & 58 & 65 & 69 & 57 & 55 & 65 & 55 \\
\hline SC15 & 70 & 97 & 77 & 69 & 85 & & 94 & 72 & 79 & 82 & 77 & 87 & 73 \\
\hline
\end{tabular}




\begin{tabular}{|l|l|l|l|l|l|l|l|l|l|l|l|l|}
\hline SC16 & 61 & 87 & 77 & 89 & 90 & 76 & 81 & 87 & 85 & 95 & 87 & 76 \\
\hline SC17 & 63 & 50 & 53 & 58 & 49 & 53 & 61 & 65 & 52 & 67 & 63 & 57 \\
\hline SC18 & 61 & 64 & 71 & 58 & 57 & 69 & 78 & 66 & 70 & 67 & 77 & 61 \\
\hline
\end{tabular}

\section{ANALYSIS AND INTEPRETATION}

In this section, we have provided the information about the analysis performed to find the relationship between metrics and quality attributes given. We employed both the univariate i.e. linear and multiple regression analysis. The univariate/linear analysis is used to find the "best" line to fit two variables so that one attribute can be used to figure out the other. Multiple linear regression is an extension of linear regression, which allows a response variable to be modelled as a linear function of two or more predictor variables

\subsection{Descriptive Statistics}

Descriptive statistics is the term given to the analysis of data that helps describe, show or summarize data in a purposeful way. Descriptive statistics are used to outline the basic features of the data in a study [7]. It provides summary of the data. The descriptive statistics of metrics are shown in Table3. The table shows the "mean", "standard deviation" "min", "max" for all metrics considered in this study.

\section{Table 3 Descriptive statistics}

\begin{tabular}{|c|c|c|c|c|}
\hline Metrics & Mean & $\begin{array}{c}\text { Standard } \\
\text { Deviation }\end{array}$ & Min & Max \\
\hline NDC & 4.94 & 1.349 & 3.00 & 8.00 \\
\hline NBC & 10.50 & 3.585 & 4.00 & 17.0 \\
\hline NC & 15.44 & 4.422 & 8.00 & 22.0 \\
\hline RBC & 2.20 & 0.802 & 1.00 & 3.40 \\
\hline NAFC & 4.22 & 2.045 & 1.00 & 10.0 \\
\hline NADC & 19.88 & 5.779 & 9.00 & 30.0 \\
\hline NABC & 16.39 & 6.652 & 6.00 & 26.0 \\
\hline NA & 40.38 & 11.067 & 21.0 & 55.0 \\
\hline NH & 3.50 & 1.098 & 2.00 & 6.00 \\
\hline DHP & 3.16 & 1.043 & 2.00 & 5.00 \\
\hline RSA & .117 & 0.0579 & .02 & .23 \\
\hline
\end{tabular}

\subsection{Correlation Analysis}

We applied Spearman's rho correlation to test the correlation between the independent (metrics) and dependent variable at the significance level=0.05 (widely used value). We evaluated the correlation among each metric with understanding time, which is an important static quantity as shown in Table4. From these results we found that understandability is correlated with NDC, NBC, NC,

NAFC, NADC , NABC, NA, NH, DHP. Significance value greater than 0.5 are highlighted in bold in Table4. The metrics RBC and RSA are not related with corresponding understanding time

Table 4 Spearman's rho correlation

\begin{tabular}{|c|c|}
\hline Metrics & Understanding Time(Correlation) \\
\hline NDC & .000 \\
\hline NBC & .000 \\
\hline NC & .000 \\
\hline RBC & .349 \\
\hline NAFC & .001 \\
\hline NADC & .029 \\
\hline NABC & .006 \\
\hline NA & .000 \\
\hline NH & .001 \\
\hline DHP & .004 \\
\hline RSA & $\mathbf{. 0 8 0}$ \\
\hline
\end{tabular}

\subsection{Univariate regression Analysis}

Univariate analysis explores each variable in a dataset separately. The analysis is carried out with the description of a single variable in terms of the applicable unit of analysis. It is concerned with the description or summarization of individual variables in a given dataset [7]. This analysis is done to examine the effect of individual metric on the dependent variable.

The results in Table 5 indicate that there is a significant relationship between NDC, NBC, NC, NAFC, NADC, NABC, NA, NH, DHP and understanding time .They are at significance level $=0.05$. The metrics having values more than significance level i.e. 0.05 are highlighted in bold in Table5. The metrics RBC and RSA are not significantly related with understanding time. From Table 5 we can observe NC metric is the most significant metric amongst all with the highest value of $F$ values i.e. 155.266. Metrics in decreasing order of $F$ values are as follows: NC NBC NA NDC NABC NAFC NADC DHP NH RBC and RSA.

Table 5 Univariate analysis of the metrics with understandability time as dependent variable

\begin{tabular}{|c|c|c|c|c|c|c|}
\hline \multicolumn{2}{|r|}{ Model } & Sum of & Degree of & Mean & $\mathbf{F}$ & Sig. \\
\hline NDC & $\begin{array}{l}\text { Regression } \\
\text { Residual } \\
\text { Total }\end{array}$ & $\begin{array}{c}594.722 \\
551.943 \\
1146.665\end{array}$ & $\begin{array}{c}1 \\
16 \\
17\end{array}$ & $\begin{array}{c}594.722 \\
34.496\end{array}$ & 17.240 & .001 \\
\hline NBC & $\begin{array}{l}\text { Regression } \\
\text { Residual } \\
\text { Total }\end{array}$ & $\begin{array}{c}935.899 \\
210.766 \\
1146.665\end{array}$ & $\begin{array}{c}1 \\
16 \\
17\end{array}$ & $\begin{array}{c}935.899 \\
13.173\end{array}$ & 71.047 & .000 \\
\hline NC & $\begin{array}{l}\text { Regression } \\
\text { Residual } \\
\text { Total }\end{array}$ & $\begin{array}{c}1039.542 \\
107.124 \\
1146.665\end{array}$ & $\begin{array}{c}1 \\
16 \\
17\end{array}$ & $\begin{array}{c}1039.542 \\
6.695\end{array}$ & 155.266 & .000 \\
\hline
\end{tabular}




\begin{tabular}{|c|c|c|c|c|c|c|}
\hline RBC & $\begin{array}{l}\text { Regression } \\
\text { Residual } \\
\text { Total }\end{array}$ & $\begin{array}{c}157.472 \\
989.193 \\
1146.665\end{array}$ & $\begin{array}{c}1 \\
16 \\
17\end{array}$ & $\begin{array}{c}157.472 \\
61.825\end{array}$ & 2.547 & .130 \\
\hline NAFC & $\begin{array}{l}\text { Regression } \\
\text { Residual } \\
\text { Total }\end{array}$ & $\begin{array}{c}536.727 \\
609.938 \\
1146.665\end{array}$ & $\begin{array}{c}1 \\
16 \\
17\end{array}$ & $\begin{array}{c}537.727 \\
38.121\end{array}$ & 14.080 & .002 \\
\hline NADC & $\begin{array}{l}\text { Regression } \\
\text { Residual } \\
\text { Total }\end{array}$ & $\begin{array}{c}315.136 \\
832.529 \\
1146.665\end{array}$ & $\begin{array}{c}1 \\
16 \\
17\end{array}$ & $\begin{array}{c}315.136 \\
51.971\end{array}$ & 6.064 & .026 \\
\hline NABC & $\begin{array}{l}\text { Regression } \\
\text { Residual } \\
\text { Total }\end{array}$ & $\begin{array}{c}554.853 \\
591.812 \\
1146.665\end{array}$ & $\begin{array}{c}1 \\
16 \\
17\end{array}$ & $\begin{array}{c}554.853 \\
36.988\end{array}$ & 15.001 & .001 \\
\hline NA & $\begin{array}{l}\text { Regression } \\
\text { Residual } \\
\text { Total }\end{array}$ & $\begin{array}{c}750.942 \\
395.723 \\
1146.665\end{array}$ & $\begin{array}{c}1 \\
16 \\
17\end{array}$ & $\begin{array}{c}750.942 \\
24.733\end{array}$ & 30.362 & .000 \\
\hline NH & $\begin{array}{l}\text { Regression } \\
\text { Residual } \\
\text { Total }\end{array}$ & $\begin{array}{c}479.741 \\
666.924 \\
1146.665\end{array}$ & $\begin{array}{c}1 \\
16 \\
17\end{array}$ & $\begin{array}{c}479.741 \\
41.683\end{array}$ & 11.509 & .004 \\
\hline DHP & $\begin{array}{l}\text { Regression } \\
\text { Residual } \\
\text { Total }\end{array}$ & $\begin{array}{c}480.913 \\
665.752 \\
1146.665 \\
\end{array}$ & $\begin{array}{c}1 \\
16 \\
17 \\
\end{array}$ & $\begin{array}{c}480.913 \\
41.610 \\
\end{array}$ & 11.558 & .004 \\
\hline RSA & $\begin{array}{l}\text { Regression } \\
\text { Residual } \\
\text { Total }\end{array}$ & $\begin{array}{c}20.414 \\
1126.251 \\
1146.665\end{array}$ & $\begin{array}{c}1 \\
16 \\
17\end{array}$ & $\begin{array}{l}20.414 \\
70.391\end{array}$ & .290 & .598 \\
\hline
\end{tabular}

Table 6 Multiple regressions for combination of two independent variables with understandability time

\begin{tabular}{|c|c|c|c|c|c|}
\hline Model & $\begin{array}{l}\text { Sum of } \\
\text { Square }\end{array}$ & $\begin{array}{c}\text { Degree of } \\
\text { freedom }\end{array}$ & $\begin{array}{c}\text { Mean } \\
\text { square }\end{array}$ & $\mathbf{F}$ & Sig. \\
\hline $\begin{array}{l}\text { NBC,NC } \\
\text { Regression } \\
\text { Residual } \\
\text { Total }\end{array}$ & $\begin{array}{l}661.030 \\
300.262 \\
961.292\end{array}$ & $\begin{array}{c}2 \\
15 \\
17\end{array}$ & $\begin{array}{c}330.515 \\
20.017\end{array}$ & 16.511 & .000 \\
\hline $\begin{array}{l}\text { NBC,NA } \\
\text { Regression } \\
\text { Residual } \\
\text { Total }\end{array}$ & $\begin{array}{l}695.166 \\
266.126 \\
961.292 \\
\end{array}$ & $\begin{array}{c}2 \\
15 \\
17\end{array}$ & $\begin{array}{c}347.583 \\
17.742 \\
\end{array}$ & 19.591 & .000 \\
\hline $\begin{array}{l}\text { NBC,NDC } \\
\text { Regression } \\
\text { Residual } \\
\text { Total }\end{array}$ & $\begin{array}{l}661.030 \\
300.262 \\
961.292 \\
\end{array}$ & $\begin{array}{c}2 \\
15 \\
17 \\
\end{array}$ & $\begin{array}{c}330.515 \\
20.017 \\
\end{array}$ & 16.511 & .000 \\
\hline $\begin{array}{l}\text { NBC,NABC } \\
\text { Regression } \\
\text { Residual } \\
\text { Total }\end{array}$ & $\begin{array}{l}672.195 \\
289.096 \\
961.292\end{array}$ & $\begin{array}{c}2 \\
15 \\
17\end{array}$ & $\begin{array}{c}336.098 \\
19.273\end{array}$ & 17.439 & .000 \\
\hline $\begin{array}{l}\text { NBC,NAFC } \\
\text { Regression } \\
\text { Residual } \\
\text { Total } \\
\end{array}$ & $\begin{array}{l}631.229 \\
330.063 \\
961.292 \\
\end{array}$ & $\begin{array}{c}2 \\
15 \\
17 \\
\end{array}$ & $\begin{array}{c}315.614 \\
22.004 \\
\end{array}$ & 14.343 & .000 \\
\hline $\begin{array}{l}\text { NBC,NADC } \\
\text { Regression } \\
\text { Residual } \\
\text { Total } \\
\end{array}$ & $\begin{array}{l}656.557 \\
304.735 \\
961.292 \\
\end{array}$ & $\begin{array}{c}2 \\
15 \\
17 \\
\end{array}$ & $\begin{array}{c}328.278 \\
20.316 \\
\end{array}$ & 16.159 & .000 \\
\hline $\begin{array}{l}\text { NBC,DHP } \\
\text { Regression } \\
\text { Residual } \\
\text { Total }\end{array}$ & $\begin{array}{l}630.314 \\
330.978 \\
961.292 \\
\end{array}$ & $\begin{array}{c}2 \\
15 \\
17 \\
\end{array}$ & $\begin{array}{c}315.157 \\
22.065\end{array}$ & 14.283 & .000 \\
\hline $\begin{array}{l}\text { NBC,NH } \\
\text { Regression } \\
\text { Residual } \\
\text { Total }\end{array}$ & $\begin{array}{l}657.076 \\
304.216 \\
961.292\end{array}$ & $\begin{array}{c}2 \\
15 \\
17\end{array}$ & $\begin{array}{c}328.538 \\
20.281\end{array}$ & 16.199 & .000 \\
\hline
\end{tabular}




\begin{tabular}{|c|c|c|c|c|c|}
\hline $\begin{array}{l}\text { NBC,RBC } \\
\text { Regression } \\
\text { Residual } \\
\text { Total }\end{array}$ & $\begin{array}{l}699.067 \\
262.224 \\
961.292\end{array}$ & $\begin{array}{c}2 \\
15 \\
17\end{array}$ & $\begin{array}{c}349.534 \\
17.482\end{array}$ & 19.994 & .000 \\
\hline $\begin{array}{l}\text { NBC,RSA } \\
\text { Regression } \\
\text { Residual } \\
\text { Total } \\
\end{array}$ & $\begin{array}{l}655.784 \\
305.507 \\
961.292 \\
\end{array}$ & $\begin{array}{c}2 \\
15 \\
17 \\
\end{array}$ & $\begin{array}{c}327.892 \\
20.367 \\
\end{array}$ & 16.099 & .000 \\
\hline
\end{tabular}

Table 7 Multiple regressions for understandability time

\begin{tabular}{|c|c|c|c|c|c|}
\hline Model & $\begin{array}{l}\text { Sum of } \\
\text { Square }\end{array}$ & $\begin{array}{c}\begin{array}{c}\text { Degree of } \\
\text { freedom }\end{array} \\
\end{array}$ & $\begin{array}{c}\text { Mean } \\
\text { square }\end{array}$ & $\mathbf{F}$ & Sig. \\
\hline $\begin{array}{l}\text { NC } \\
\text { Regression } \\
\text { Residual } \\
\text { Total }\end{array}$ & $\begin{array}{c}1039.542 \\
107.124 \\
1146.665\end{array}$ & $\begin{array}{c}1 \\
16 \\
17\end{array}$ & $\begin{array}{c}1039.542 \\
6.695\end{array}$ & 155.266 & .000 \\
\hline $\begin{array}{l}\text { NC,NBC } \\
\text { Regression } \\
\text { Residual } \\
\text { Total } \\
\end{array}$ & $\begin{array}{c}1043.306 \\
103.359 \\
1146.665 \\
\end{array}$ & $\begin{array}{c}2 \\
15 \\
17 \\
\end{array}$ & $\begin{array}{c}521.653 \\
6.891 \\
\end{array}$ & 75.705 & .000 \\
\hline $\begin{array}{l}\text { NC,NBC,NA } \\
\text { Regression } \\
\text { Residual } \\
\text { Total } \\
\end{array}$ & $\begin{array}{c}1046.071 \\
100.594 \\
1146.655 \\
\end{array}$ & $\begin{array}{c}3 \\
14 \\
17\end{array}$ & $\begin{array}{c}348.690 \\
7.185 \\
\end{array}$ & 48.528 & .000 \\
\hline $\begin{array}{l}\text { NC,NBC,NA,NDC } \\
\text { Regression } \\
\text { Residual } \\
\text { Total }\end{array}$ & $\begin{array}{c}1046.071 \\
100.594 \\
1146.665 \\
\end{array}$ & $\begin{array}{c}3 \\
14 \\
17 \\
\end{array}$ & $\begin{array}{c}348.690 \\
7.185 \\
\end{array}$ & 48.528 & .000 \\
\hline $\begin{array}{l}\text { NC,NBC,NA NDC,NABC } \\
\text { Regression } \\
\text { Residual } \\
\text { Total } \\
\end{array}$ & $\begin{array}{c}1062.035 \\
84.630 \\
1146.665 \\
\end{array}$ & $\begin{array}{c}4 \\
13 \\
17 \\
\end{array}$ & $\begin{array}{c}265.509 \\
6.510 \\
\end{array}$ & 40.785 & .000 \\
\hline $\begin{array}{l}\text { NC,NBC,NA,NDC,NABC,NAFC } \\
\text { Regression } \\
\text { Residual } \\
\text { Total } \\
\end{array}$ & $\begin{array}{c}1063.133 \\
83.532 \\
1146.665 \\
\end{array}$ & $\begin{array}{c}5 \\
12 \\
17 \\
\end{array}$ & $\begin{array}{c}212.627 \\
6.961 \\
\end{array}$ & 30.545 & .000 \\
\hline $\begin{array}{l}\text { NC,NBC,NA,NDC,NABC,NAFC,NADC } \\
\text { Regression } \\
\text { Residual } \\
\text { Total } \\
\end{array}$ & $\begin{array}{c}1075.727 \\
70.938 \\
1146.665 \\
\end{array}$ & $\begin{array}{c}6 \\
11 \\
17 \\
\end{array}$ & $\begin{array}{c}179.288 \\
6.449 \\
\end{array}$ & 27.801 & .000 \\
\hline $\begin{array}{l}\text { NC,NBC,NA,NDC,NABC,NAFC,NADC,DHP } \\
\text { Regression } \\
\text { Residual } \\
\text { Total }\end{array}$ & $\begin{array}{c}1075.728 \\
70.937 \\
1146.665 \\
\end{array}$ & $\begin{array}{c}7 \\
10 \\
17\end{array}$ & $\begin{array}{c}153.675 \\
7.094\end{array}$ & 21.664 & .000 \\
\hline $\begin{array}{l}\text { NC,NBC,NA,NDC,NABC,NAFC,NADC,DHP,NH } \\
\text { Regression } \\
\text { Residual } \\
\text { Total }\end{array}$ & $\begin{array}{c}1077.440 \\
69.225 \\
1146.665 \\
\end{array}$ & $\begin{array}{c}8 \\
9 \\
17 \\
\end{array}$ & $\begin{array}{c}134.680 \\
7.692 \\
\end{array}$ & 17.510 & .000 \\
\hline $\begin{array}{l}\text { NC,NBC,NA,NDC,NABC,NAFC,NADC,DHP,NH,RBC } \\
\text { Regression } \\
\text { Residual } \\
\text { Total }\end{array}$ & $\begin{array}{c}1096.193 \\
50.472 \\
1146.665 \\
\end{array}$ & $\begin{array}{c}9 \\
8 \\
17\end{array}$ & $\begin{array}{c}121.799 \\
6.309 \\
\end{array}$ & 19.306 & 0.00 \\
\hline $\begin{array}{l}\text { NC,NBC,NA,NDC,NABC,NAFC,NADC,DHP,NH,RBC,RSA } \\
\text { Regression } \\
\text { Residual } \\
\text { Total }\end{array}$ & $\begin{array}{c}1102.189 \\
44.476 \\
1146.665 \\
\end{array}$ & $\begin{array}{c}10 \\
7 \\
17\end{array}$ & $\begin{array}{c}110.219 \\
6.354 \\
\end{array}$ & 17.347 & .001 \\
\hline
\end{tabular}

Table 8 Model summary of understanding time

\begin{tabular}{|l|l|l|l|}
\hline Model & $\mathbf{R}$ & $\mathbf{R}^{\mathbf{2}}$ & Adjusted $^{\mathbf{2}}$ \\
\hline NC & .952 & .907 & .901 \\
\hline NC,NBC & .954 & .910 & .898 \\
\hline NC,NBC,NA & .955 & .912 & .893 \\
\hline NC,NBC,NA,NDC & .955 & .912 & .893 \\
\hline NC,NBC,NA,NDC,NABC & .962 & .926 & .903 \\
\hline NC,NBC,NA,NDC,NABC,NAFC & .963 & .927 & .897 \\
\hline NC,NBC,NA,NDC,NABC,NAFC,NADC & .969 & .938 & .904 \\
\hline
\end{tabular}




\begin{tabular}{l}
\hline NC,NBC,NA,NDC,NABC,NAFC,NADC,DHP \\
\hline NC,NBC,NA,NDC,NABC,NAFC,NADC,DHP,NH \\
\hline NC,NBC,NA,NDC,NABC,NAFC,NADC,DHP,NH,RBC \\
\hline NC,NBC,NA,NDC,NABC,NAFC,NADC,DHP,NH,RBC,RSA \\
5.4 Multiple regressions Analysis \\
Multiple regressions allow us to predict one response variable \\
using two or more predictor variables. The general motive of \\
multiple regressions is to learn more about the relationship \\
between several independent variables and a dependent \\
variable. This technique looks at the relationship between \\
independent and dependent variables and also considers the \\
former in combinations, in order to best explain the variance \\
of dependent variable due to independent variables and finally \\
derive predictions.
\end{tabular}

In this section the results of multiple regressions to see the effect of various combinations of independent variables on the dependent variable i.e. understanding are presented. [8]Table 6 represents multiple regressions for combination of two independent variables with understandability time. It is noticed that for each model i.e. every combination of two metrics has noteworthy relation with the output variable i.e. understanding time.

In a similar way, we find the multiple regressions by combining every permutation of metrics. We have displayed the final results of the multiple regressions which are strongly related to understanding time i.e. we have combined the metrics in the descending order of $\mathrm{F}$ values given in Table 5 . Combination of metrics in the descending order of $F$ values is shown in Table 7.

Now, according to the combination of metrics given in Table 7 , we found the value of $R, R^{2}$ and adjusted $R^{2}$. Here, $R$ is the sample coefficient used for linear regression. $R^{2}$ is the coefficient of determination which gives information about the goodness of fit of a model. In regression, the $\mathrm{R}^{2}$ is a statistical measure of how well the regression line approximates the real data points. An $\mathrm{R}^{2}$ of 1.0 indicates that the regression line perfectly fits the data. Adjusted $\mathrm{R}^{2}$ is a

modification of $\mathrm{R}^{2}$ that adjusts for the number of explanatory terms in a model.

Table 8 represents the regression model summary of understanding time. It shows the variance explained due to independent variables by observing value of $\mathrm{R}^{2}$. We observed the change in variance when these metrics are combined. We started with $\mathrm{NC}$ as it explains maximum variance in the dependent variable as compared to other metrics. Table 8 represents the final results. It shows that in the final model value of adjusted $\mathrm{R}^{2}$ is .906 i.e. $90.6 \%$ of independent variables have been covered, which indicates a considerably good quality model .We have also observed that change in variance explained due to NDC is nil, hence NDC does not have a significant effect on understandability.

\section{THREATS}

This section discusses about the threats to the validity of results and limitations. As we know, several types of threats to the validity of results of an experiment exist. In this section we talk about threats to construct, internal, and conclusion validity. To assure construct validity, it is necessary to perform more experiments. For internal validity, all the subjects possess same degree of experience in data warehouse and modelling. Subjects have never performed this kind of

\begin{tabular}{|l|l|l|}
\hline .969 & .938 & .895 \\
.969 & .940 & .886 \\
\hline .978 & .956 & .906 \\
\hline .980 & .961 & .906 \\
\hline
\end{tabular}

experiment in past, so in our case persistent effects are not present. For conclusion validity, we have taken a sample value of 18 , which may not be enough for statistical test. We may attempt a bigger data set through more experimentation.

In this paper we are focusing on two quality factors, i.e. understandability and efficiency. One of the limitations of this work is the size of data. Though we performed this experiment with more numbers of schemas as compared to earlier conducted study [15], but we still feel that more data is required to deal with conclusion validity regarding the effect of metrics on quality attributes. Another limitation of this work is that we have conducted this experiment by considering students as subjects. Due to difficulty in getting professionals to perform the experiment, this experiment was done by students.

\section{CONCLUSION}

In this paper, we have carried out empirical validation of metrics using statistical techniques. The systems under study were eighteen schemas. In this paper, initially we observed a correlation between the metrics and understandability and subsequently found the effect of various combinations of metrics using univariate and multiple regression techniques of statistics. The results in Table 8 displays that metrics have a noteworthy effect on the quality attribute -understandability.

As a future work, it is essential to keep on performing empirical validation exercises by considering professional subjects, crafting new experiments with more cases and different values of metrics, running case studies with real data from industrial environment to ensure conclusion validity. These experiments will assist data warehouse users in many processes of data warehousing. Thus will help in improving the overall development process of a datawarehouse.

\section{REFERENCES}

[1] V.R. Basili, F. Shull and F. Lanubille. Building Knowledge through families of experiments. IEEE Transactions on Software Engineering.No. 4.456-473, July/August, 1999.

[2] L.C. Briand, S. Morasca and V. Basili. Propertybased software engineering measurement. IEEE Transactions on Software Engineering. 22(1).68-85, 1996.

[3] Calero C., Piattini M., Pascual C., Serrano, M.A. (2001), "Towards Data warehouse quality metrics.In $3^{\text {rd }}$ International workshop on design and Management of Data warehouses (DMDW 2001), Interlaken, Switzerland.

[4] Inmon,, W. H. (1997), "Building Data warehouse”, John Wiley \& sons.

[5] Fenton N. (1994), "Software measurement: a necessary scientific basis," IEEE Transactions on Software Engineering, Vol. 20, 1994, pp. 199-206.

[6] GARDNER, S.R.: 'Building the data warehouse', Comnzun. ACM, September 1998,41, (9), pp. 52-60.

[7] Gupta SL, Kumar V (2011) Statistical mechanics. Pragati prakashan, Meeru [GS11] Gosain A, Nagpal S, Sabharwal S, "Quality Metrics for Conceptual Models 
for Data Warehouse focusing on Dimension Hierarchies" July 2011 ACM SIGSOFT.

[8] Gosain A, Mann S (2013) 'Empirical validation of metrics for object oriented multidimensional model for data warehouse', Springer Int J Syst Assur Eng Manag.

[9] Gosain A, Nagpal S, Sabharwal S, Validating dimension hierarchy metrics for the understandability of multidimensional models for data warehouse.

[10] R. Kimball, L. Reeves, M. Ross and W.Thornthwaite. The Data Warehouse LifecycleToolkit, John Wiley and Sons, 1998.

[11] Kumar M, Gosain A, Singh Y, Empirical validation of structural metrics for predicting understandability of conceptual schemas for data warehouse.

[12] Serrano M., Calero C., Piattini M. (2002), "Validating metrics for data warehouses", IEE Proceedings SOFTWARE 149, 161-166.

[13] Serrano M, Calero M, Piattini M (2003) 'Experimental Validation of Multidimensional Data Models Metrics",
IEEE Proceedings of the 36th Hawaii International Conference on System Sciences - 2003.

[14] Serrano MA, Calero C, Trujillo J, Lujan S, Piattini M (2004) "Empirical validation of metrics for conceptual models of data warehouse." Lecture Notes Comput Sci 3084:506-520 (Caise2004).

[15] Serrano M., Trujillo j, Calero C., Piattini M. (2007), "Metrics for data warehouse conceptual models understandability",Journal of Information and Software Technology49, 851-870.

[16] Serrano M, Calero C, Sahraoui HA, Piattini M (2008) 'Empirical studies to assess the understandability of data warehouse schemas using structural metrics." Softw Qual J Springer 16:79-106.

[17] E.J. Weyuker. Evaluating software complexity measures. IEEE Transactions on Software Engineering. 14(9). 1357-1365, 1988.

[18] H. Zuse. A Framework of SoftwareMeasurement. Walter de Gruyter, 1998 